\title{
Globally and locally applied naturalistic metaphysics*
}

\section{Cristian Soto}

\author{
Universidad de Chile \\ Department of Philosophy \\ Nuñoa \\ Chile \\ cssotto@gmail.com
}

\section{Article info}

CDD: 110

Received: 10.06.2017; Revised: 02.10.2017; Accepted: 02.10.2017

DOI: http://dx.doi.org/10.1590/0100-6045.2017.V40N3.CS

\author{
Keywords: \\ Naturalistic metaphysics \\ Scientific image \\ Applicability \\ Ontology \\ Unobservable entities \\ Sellars
}

\begin{abstract}
This article addresses the prospects of applied naturalistic metaphysics from both a global and a local perspective. Adopting a broad Sellarsian approach, I look into whether metaphysics has a place and role in the overall scientific image and assess whether it has its own subject matter as a first- or second- order discipline. After outlining the general argument in section 1, section 2 examines our construal of science and metaphysics, drawing some considerations for restating the question about the viability of naturalistic metaphysics. Sections 3 and 4, in turn, suggest two styles of naturalistic metaphysics that can be respectively applied on a global and on a local basis. I briefly outline their respective goals, problems and categories. I argue, in particular, that globally applied naturalistic metaphysics deals with issues about the fundamental structure of reality, whereas locally applied naturalistic metaphysics tackles riddles arising from the examination of specific unobservable posits in the frontiers of scientific ontology. Section 5 closes with concluding remarks that put metaphysics within the scientific image.
\end{abstract}

\footnotetext{
* This article is a result of the author's governmental funded research grant FONDECYT de Iniciación No. 11160324, "The Physico-Mathematical Structure of Scientific Laws," CONICYT, Chile.
} 


\section{Introductory remarks: the Sellarsian prospect of a scientific image}

The goal of this paper consists in examining the prospects of applied naturalistic metaphysics in view of both global and local concerns arising from scientific ontology. I shall suggest a distinction between globally and locally applied (hereafter, GA and LA, respectively) naturalistic metaphysics. In section 2, I pay close attention to our construal of science and metaphysics. Although I do not intend to claim that the distinction is clear-cut, my hope is that it provides us with a better grasp of metaphysical practices. I contend that the question about the viability of applied naturalistic metaphysics does not have to do with whether we can blend metaphysics in the sciences, as if the former had to imitate the latter. Instead, the viability of the discipline depends upon whether it succeeds to make global and local contributions to the articulation of a scientific worldview in our overall intellectual endeavour.

When suggesting the distinction between GA and LA naturalistic metaphysics in sections 3 and 4, respectively, I shall assume that metaphysics largely is an a priori enterprise by its own nature. Here are some preliminary definitions. On the one hand, $G A$ naturalistic metaphysics is the branch of metaphysical practice that aims at investigating ontological issues stemming from the fundamental structure of reality, i.e., those features of the physical world that are expected to be instantiated everywhere in the universe. Examples of such problems are those of whether reality has a natural-kind structure, whether space and time are substantival or merely relational, whether there ultimately is (causal) order in the world, and so forth.

On the other hand, $L A$ naturalistic metaphysics deals with specific conundrums resulting from the examination of specific theories in the frontiers of scientific ontology, where the postulation of particular unobservable posits appears essential to articulate a thorough scientific worldview. Issues of the latter sort are generally framed upon concerns regarding the ontological status of unobservable entities, especially when some form or another of inference to the best explanation comes to play. I shall specifically focus on the postulation of one unobservable scientific posit (or cluster of them), namely: dark matter.

Some may wonder why such problems as the fundamental structure of reality and the postulation of unobservable entities involve any metaphysics whatsoever. It should be observed that my distinction between the two forms of metaphysical practices sides with the naturalistic trend in current debates, which holds the view that if metaphysics is worth pursuing, it needs to work on a par with the sciences

Manuscrito - Rev. Int. Fil. Campinas, v. 40, n. 3, pp. 33-50, jul.-set. 2017. 
in the investigation of the physical world (see Ladyman et al. 2007, Morganti 2013, and Livanios 2017, among others). I have undertaken this line of argument elsewhere (Soto 2015), demonstrating that the relationship between scientific and metaphysical practices is one in which the former needs the latter in order to tackle issues concerning the articulation of a scientific worldview, while at the same time the latter is fuelled by the former when addressing riddles related to the fundamental structure of the world and the ontological status of unobservable posits in the frontiers of scientific ontology. For my argument to work, I need to set forth reasons to think that metaphysics has both global and local applications to the scientific endeavour, contributing to shaping in different ways our science-based conception of reality.

My reading of the problem of the possibility of naturalistic metaphysics is partially motivated by Sellars' defence of the scientific image. In a Sellarsian spirit, I argue that GA and LA naturalistic metaphysics have their own place and role in our investigation of the physical realm, thereby accommodating to the scientific image (Sellars 1963, pp. 18 and ff.). However, in due course, I shall highlight certain points where my views take distance from those of Sellars'. In section 5, I refer to the know how/know that distinction (Sellars 1963, pp. 1-3) in order to raise the question of whether GA and LA naturalistic metaphysics are first- or second-order disciplines in view of their subject matters. In this regard, I maintain that even though both GA and the LA metaphysical practices can be conceived of as second-order disciplines reflecting on first-order scientific theorising, this does not pose a threat to the disciplinary independence of metaphysical practice itself, nor does it cast a doubt on its place and role in the orders of knowledge.

\section{Restating the question: construals of science and metaphysics}

The question about the viability of naturalistic metaphysics has been differently stated in recent debates in both the philosophy of science and analytic metaphysics (see Ladyman 2007 and 2012; Ney 2012; and Ross 2012; among others). The problem at stake involves several assumptions. Overall, it is granted that both the sciences and metaphysics aim at yielding knowledge and understanding of the material world (see Cumpa 2014, pp. 320 and 322), whereas at the same time it is commonly agreed that the epistemic successes of the sciences far surpass those of metaphysics in accomplishing such tasks. As a result, 
metaphysicians are challenged to justify in the first place, as it were, the worthiness of their own activity. Granted that we have a successful, institutionally organized physico-mathematical scientific enterprise, metaphysicians need to come up with a reason for us to believe that we can still make good use of metaphysical theorising when finding out about the physical realm.

The intricacies of the problem about the viability of naturalistic metaphysics have led philosophers to interpret the challenge in many ways. A naïve reading of the question focuses on whether we should expect naturalistic metaphysics to imitate the sciences, getting as close as possible to the methodologies and epistemic standards of scientific practice. According to this, naturalistic metaphysics would be required to employ mathematics in the formulation of its theories, to recur to experimental techniques to collect data, and to follow patterns of generation and testing of hypotheses similar to those of the sciences (Bunge 1972). Nevertheless, by its own nature, metaphysics cannot but fail to respond to such expectations. As a result, the naïve reading inevitably leads to the adoption of one form or another of eliminativism about metaphysics (van Fraassen 2002, chapter 1). Advocates of the latter view are sceptics about attributing metaphysics a place and role in the scientific image. Moreover, in their view, the sole epistemic successes of the sciences should suffice for us to dispense with metaphysical theorising altogether.

The naïve reading, however, misconstrues the problem. It gets things wrong when it assumes that for metaphysics to be naturalized it has to respond to the methodologies and epistemic standards of the sciences. Contrary to this, there is room for arguing that if metaphysical practice is deemed to perform a naturalistic turn, it has to do so as a discipline that can positively contribute to shaping our scientific worldview, but on its own grounds.

Let us articulate a sophisticated reading of the problem. The first issue to take up at this point is related to how we should understand both the sciences and metaphysics. This is because it is not always clear the way in which we should conceive of scientific practice when thinking about the viability of naturalistic metaphysics. A narrow construal of the sciences tells us that scientific communities are in the business of collecting data about the world by means of experiments and observations, laying the grounds for applying mathematics to 
the construction of theories and models that convey explanations and predictions of relevant phenomena (Morganti 2013, p. 8). ${ }^{1}$

We can broaden this characterisation adding the social dimension of science, which highlights the fact that the various branches of science are the result of complex processes of institutionalisation, which are oriented to establish error filters that guarantee the epistemic reliability of theories about the way the world is (Ladyman et al. 2007, pp. 37 and 57). Importantly, the resulting broad construal of the sciences allows us to remain reasonably pluralistic about the diversity of our epistemic interactions with the world. To be sure, institutionalization processes of scientific disciplines take place in view of domain-specific subject matters. We find striking methodological differences even among standard scientific disciplines. Manipulation of phenomena may be relevant in experimental physics, chemistry and molecular biology, but not as much so in scientific cosmology; phenomena in economics instantiate kinds of processes which are not easily comparable to those of quantum world; entities routinely included in our scientific image go from concrete physical posits to the abstract realm of the mathematicalia partaking in representations of ideal systems; and so forth. Scientific knowledge -and for that matter, human knowledge in generaldoes not follow a uniquely, single-driven path, as though it were led by a caricature of a lineal, all-encompassing enterprise.

A first element for articulating a sophisticated reading of our main concern consists in extending to metaphysics this pluralistic stance on our epistemic interactions with the world that underpins scientific agenda. Such epistemic pluralism opens the possibility for metaphysical practice to positively contribute to our intellectual endeavour. Recall that naturalistic metaphysics is largely a priori, even though it is concerned with the physical world. The challenge for metaphysicians is to show that metaphysical practice has its own methodologies and epistemic standards, which enable it to provide knowledge or understanding

\footnotetext{
${ }^{1}$ I thank one of the Manuscrito's referees for pointing out to me that the narrow construal of science leaves to one side non-empirical sciences. In particular, the case of mathematics may challenge the narrow construal. I address relevant comparisons between pure mathematics and metaphysics in section 3.2 below. At present, the narrow construal, which I only sketch to highlight the scope of my argument in what follows, is to be restricted to empirical sciences only.
} 
of its own topics. If that can be done, there is no reason to deny metaphysics its own place and role in the scientific image along with the rest of the sciences. ${ }^{2}$

Here is yet a second element for our construal of metaphysics, namely: metaphysical practice is not a homogeneous discipline. By contrast, it comes in different flavours. Sections 3 and 4 shall suggest distinguishing between $G A$ and $L A$ naturalistic metaphysics, respectively. The distinction aims to characterise two ways in which metaphysical practice has been somehow institutionalized, each one with its own scope, problems and categories, contributing in their own ways to shaping our scientific image of the world.

Let us close this section restating our main question as follows. The problem about the prospects of naturalistic metaphysics asks for the place and role of metaphysical research in our overall intellectual endeavour, along with the rest of the sciences. If we want to set forth a positive response, we need to show that both GA and LA naturalistic metaphysics succeed in yielding knowledge or understanding of their respective subject matters of enquiry. I will provide two examples demonstrating that this is indeed the case. After describing in further detail GA naturalistic metaphysics in section 3, I shall draw on Ladyman and Ross' (2013) ontic structural realism in order to provide an example of global application at work. In turn -as mentioned above-, section 4 addresses the ontological status of dark matter in astrophysics, claiming that the ontological riddles this entity involves can be illuminated from the perspective of local applications.

\section{GA naturalistic metaphysics}

\subsection{Outlining the global approach}

In order not to beg the question against GA naturalistic metaphysics, we need to accord it, in the first place, its own methodologies and epistemic standards institutionally agreed upon by the discipline and for itself. GA naturalistic

\footnotetext{
${ }^{2}$ I am aware that philosophers of science whose views are rooted in the positivist tradition will by no means agree with the sort of epistemic pluralism I suggest. They, by contrast, would declare themselves in favour of the monolithic methodological rule that states that the correct methodologies of enquiring into the nature of reality are exclusive of the physical sciences, most likely of experimental physics (see van Fraassen 2002).
} 
metaphysics has its own scope, namely: it aims at investigating the fundamental structure of reality. As such, it employs a priori epistemic tools, such as conceptual analysis, intuitions and idealizations of various sorts. Its own practices safeguard this style of metaphysics, especially since it displays a more or less welldefined set of concepts such as natural kinds, universals, properties, and dispositions, among others, which are usually employed by its practitioners in categorising aspects of reality. Granted this, we ask: does GA naturalistic metaphysics have its place and role in the scientific image?

\subsection{A comparison with pure mathematics}

Some suggest a comparison between GA naturalistic metaphysics and pure mathematics when defending the place and role of the former in human knowledge (see Watkins 1975, p. 91; Morganti 2013, p. 1). ${ }^{3}$ We can articulate the comparison in the following terms. Both GA naturalistic metaphysics and pure mathematics proceed by means of pure thought, recurring to conceptual analysis, intuitions, idealizations, and so forth. What is more, although disconnected from the empirical sciences, pure mathematics has been successfully carried out for over 2500 years, demonstrating progression in the generation and solution of several mathematical puzzles and in the refinement of our expertise of the mathematical domain. Similarly, or so the story goes on, a parallel case can be made for GA naturalistic metaphysics. The latter has been practiced for over 25 centuries, and certain progression can be observed in its development: think of the early a priori metaphysical speculations about atomism in the hands of Democritus and Lucretius, or the early a priori metaphysical speculations about causation by Aristotle. Both problems have experienced refinement over the years.

Be that as it may, the comparison between GA naturalistic metaphysics and pure mathematics soon falls apart since there are some patent differences between them. First, we find a substantial dissimilarity in what it means for the disciplines in question to be pure and to be a priori. Pure mathematics is just not concerned with the physical realm; not only is it detached from the empirical

\footnotetext{
${ }^{3}$ Note that this section focuses strictly on pure mathematics. The question I address has to do with whether metaphysics can be pure in the sense that large parcels of mathematics are pure. As the acronym $G A$ shows, I reject this possibility and grant that metaphysics, even in its global fashion, is to be concerned with the physical world.
}

Manuscrito - Rev. Int. Fil. Campinas, v. 40, n. 3, pp. 33-50, jul.-set. 2017. 
sciences, but it is not in principle interested in putative applications. By contrast, the situation in this respect is radically different when it comes to GA naturalistic metaphysics, since we should expect even the most abstract, a priori reasoning in GA naturalistic metaphysics to have some bearing on reality. Furthermore, any form of pure metaphysics, so to speak, which is not concerned with reality at all, risks becoming a mere whim with no epistemic purport whatsoever. ${ }^{4}$

Another difference is this: even though pure mathematics is in principle detached from empirical investigation and is not directly concerned with finding out truths about the physical world, it has led to many surprising applications to scientific theorising. In particular, unforeseen applications to physics (such as the use of Hilbert spaces in quantum mechanics; the contribution of Minkovskian geometry to the theory of general relativity; and Dirac's relativistic wave equation suggesting the reality of antimatter) speak in favour of the powerful epistemic purport of pure mathematical theorising when it gets to be applied to physics. A common feature of such cases is that pure mathematics has truly led to impressive progresses in uncovering features of physical systems. Those advocating the comparison between pure mathematics and GA naturalistic metaphysics are thereby challenged to provide a story about cases in which theories in this branch of metaphysics have accomplished alike contributions to the empirical sciences. It should be revealed, for instance, that the language of metaphysics is as useful for the articulation of scientific theories as mathematical language is, conveying a similar epistemic contribution; that purely speculative metaphysical theories have come to stimulate progress in physical theorising; and so forth. As far as I see, there is no easy way to provide such a story. ${ }^{5}$

\footnotetext{
${ }^{4}$ One of the referees for Manuscrito interestingly pushes on this comparison asking what would happen with the metaphysics of numbers, especially since -for some- such branch of metaphysics would not deal with entities or structures in nature. Although I cannot tackle this issue, I should mention that my views on both GA and LA naturalistic metaphysics are compatible with an agnostic stance on the reality of mathematicalia. I have argued so elsewhere (Soto, forthcoming)

${ }^{5}$ Further distinctions can be highlighted. Humphreys (2013) points out a third difference between GA naturalistic metaphysics and pure mathematics, arguing that the latter, but not the former, finds in its toolbox a precise nomenclature and rules of reasoning.
} 


\subsection{Global application and understanding}

At this stage of the argument, we still need to show that the methodologies and epistemic standards of GA naturalistic metaphysics are a source of information about reality. Rather than comparing this branch of metaphysics with pure mathematics, we can look into what global applicability amounts to in practice. More to the point, we can ask: Does GA naturalistic metaphysics provide knowledge of its subject matter? Or, does it instead yield understanding of global features of the physical realm?

Let us draw a distinction between knowledge and understanding in the following terms. Empirical sciences convey knowledge of their subject matter so long as they make possible a robust epistemic access to their targets by means of detections or measurement procedures, allowing us to refine such access to them and track their features in space and time, where such tracking is counterfactually dependent on the object, i.e., if the target were not there, we would not have access to it (see Azzouni 2004, p. 129; and Bueno 2011, p. 98). This is a broadly empiricist conception of scientific knowledge, and it best accounts for the minimal epistemic standards expected to be employed across the diversity of empirical scientific disciplines. However, as Sellars (1963, p. 3) suggested in his own way, knowledge in this sense is to be demarcated from understanding. Whereas scientific disciplines yield first-order knowledge of their targets, GA naturalistic metaphysics appears restricted to convey a suitable interpretation of the ultimate ontological assumptions involved, in this case, in fundamental physical theorising, thereby aiding a better grasp of the scope of such area of knowledge.

Let me develop the following example: if we accept that quantum mechanics currently is the best first-order description we have of the fundamental structure of reality, then we should expect GA naturalistic metaphysics to provide a second-order metaphysical framework that enables us to explore the ontological presuppositions of quantum mechanics concerning the constitution of the fundamental stuff making up reality. Allow me to refer to ontic structural realism (for short, OSR) and the way it approaches the metaphysics of quantum mechanics. OSR's metaphysics is inspired by Peirce's ideas on hypothesis, laws of nature and psychophysics (Ladyman and Ross 2013, pp. 142-148). Peirce's notion of hypothesis is interpreted as a procedure that yields explanation and qualitative amplification of knowledge. More specifically, the notion of hypothesis is conceived of as a generalisation worthy of further investigation 
because it structures ontologies of sample-generating processes, in view of which scientists compute frequency distribution of variables that they want to predict or control. As to the second element, laws of nature are understood in Peirce's view as part of a permanent structural change underway in reality. Insofar as laws of nature evolve from chance, their constants of reference are not fixed, but evolving. Indeed, the access we have to them is in relevant cases statistical and reveals the stochastic character of reality. Lastly, with respect to the third element, Peirce's interest in psychophysics encouraged him to reinterpret properties of frequencies not as second-order properties of judgments, but instead as basic properties of reality that constitute its structure. In short, the Peircean framework, along with an up-to-date scientific and philosophical knowledge of quantum mechanics, allows OSR to put forward their view of reality as irreducibly stochastic, namely: the world is the totality of nonredundant statistical data, namely the endless wave of patterns that science will go on uncovering for as long as scientific research is pursued.

In brief, OSR advances a conception of reality as being irreducibly stochastic. In so doing, it intends to meet metaphysical challenges currently resulting from the examination of this branch of science, holding the idea that quantum mechanics is the only mature part of science which is reasonably intended to restrict all possible measurement values in the universe at all scales (Ladyman and Ross 2013, pp. 131-132). Hence, OSR is designed to deal with quantum mechanical conundrums such as systems in superposition with respect to observables and the measurement problem. ${ }^{6}$

Hence, as to the question of whether GA naturalistic metaphysics has its place and role in our overall intellectual endeavour, we can answer in the positive, at least in the sense that it aims in principle to deliver understanding of our scientific worldview. More specifically, it sheds further light upon ontological

\footnotetext{
${ }^{6}$ It may be wondered whether the metaphysics of OSR does indeed amount to an example of GA naturalistic metaphysics. Ladyman and Ross' (2013, pp. 108 and ff.) contribution adds new elements to previous versions of OSR (see Ladyman et al. 2007). In particular, as an example of GA naturalistic metaphysics, OSR tackles ontological issues arising from the quantum mechanics, which tells us that that reality appears to be ultimately stochastic in nature. To be sure, the metaphysics of OSR is to be conceived of as a specific alternative for making sense of the way in which quantum mechanics describes the world.
} 
presuppositions in scientific ontology introduced in fundamental physical theorising.

Additionally, the Sellarsian distinction between first- and second-order disciplines invites a further concern that has to do with how we should interpret the interaction between GA naturalistic metaphysics and fundamental disciplines in science. Is there a dependence relationship of the former upon the latter? I shall return to this issue in sections 4 and 5 . Our construal of GA naturalistic metaphysics inclines us to attribute this form of metaphysical practice a place and role in our scientific image so long as it is duly informed by current best scientific theorising. ${ }^{7}$

\section{LA naturalistic metaphysics}

\subsection{Outlining the local approach}

I understand LA naturalistic metaphysics as the form of metaphysical practice that deals with issues in the frontiers of scientific ontology. It is local since its subject matter encompasses ontological riddles arising from specific parts of scientific theories that postulate unobservable posits whose ontological status cannot be decided by empirical evidence alone at a given time. The emphasis on specific parts of theories responds to the fact that issues of interest for LA naturalistic metaphysics emerge from the postulation of single entities or their properties. Current examples of this sort are unobservable scientific posits such as dark matter in astrophysics - which is the case study I will address below-, quarks in the standard model of particle physics, and so forth others. Although these examples are related to posits that routinely take place in the formulation of current best scientific theories in their respective domains, they are the subject matter of ontological concern for various recent developments in LA naturalistic metaphysics.

The local approach embraces the overall a priori character of metaphysical practice. Certainly, even though practitioners of this style of naturalistic metaphysics need to be informed by current best theories in their scientific domains of interest, their work does not primarily consist in gathering data or

\footnotetext{
${ }^{7}$ For further examination of the asymmetrical relationship between GA naturalistic metaphysics and fundamental scientific theories, see Esfeld (2007) and Maudlin 2007.
} 
applying mathematics to the construction of theories and models. By contrast, they apply a priori reasoning locally to those cases where we reach the frontiers of scientific ontology and deal with posits regarding which knowledge in the empiricist sense abovementioned is not available, that is, cases where we lack robust epistemic access to them, we fail to track them in space and time, or we are unable to detect them by means of observation or experimentation.

\subsection{Cashing out the continuity thesis locally}

LA naturalistic metaphysics constitutes a second-order discipline that reflects on unobservable posits involved in scientific theories. As such, it fits Sellars' (1963, pp. 1, 3 and 20) characterisation of metaphysics. As in the case of the global approach, LA naturalistic metaphysics relies in the continuity of scientific and metaphysical investigations. Such continuity is reflected in the interaction between LA naturalistic metaphysics and the sciences, where the former appears to depend upon the latter, given that LA naturalistic metaphysics takes particular scientific theories to be the source of both information and problems concerning the ontological status of unobservable posits. Philosophers in this trend may dissect, so to speak, parcels of scientific theorising in order to evaluate what entities we should take scientists to really believe in.

As may be expected, there is no general agreement about the way in which the continuity thesis should be interpreted, let alone from the local perspective. Various proposals are currently in offer. One alternative, which can straightforwardly be related to our construal of LA naturalistic metaphysics, consists in claiming that science is inherently metaphysical insofar as it involves substantial parcels of theorising about the unobservable realm (see Chakravartty 2010, p. 62). This invites us to identify unobservable scientific posits with unobservable metaphysical posits. Pursuing this argument, Chakravartty (2013) has recently elaborated two criteria designed to evaluate the epistemic purport of metaphysical theorising in terms of its proximity to empirical investigation, namely: experiential distance and (dis-)confirmational risk. I shall refer to such criteria below in further detail when assessing the case study of the postulation of dark matter in astrophysics. 


\subsection{An example of the frontier of scientific ontology: dark matter}

In this section, I examine whether the identification of scientific and metaphysical unobservable posits can be put at work from the local perspective, looking into the positing of dark matter in astrophysics, the restrictions of our epistemic access to it, and what it teaches us about the contribution of LA naturalistic metaphysics to the examination of the ontological status of unobservable entities more generally. Overall, the present case study should lead us to show that metaphysical analysis can help us assess the ontological status of unobservable posits in science.

Dark matter is thus far an unobservable entity that plays a central explanatory role in astrophysics, an area that has brought together investigations in both scientific cosmology and particle physics. As early as the 1930s, astrophysicists begun to suspect that there was some missing matter in their descriptions of the dynamical behaviour of galaxies. The main discrepancy arose at the time by comparing the observed mass of galaxies and the total mass required for explaining their gravitational effects. In the 1970s, new computational and observational techniques facilitated the establishment of the discrepancy as real. That was an important step, since it proved that the incongruity between data and theory was not merely mathematical. Nevertheless, no accurate description of the nature of dark matter was available. By contrast, several theoretical candidates were proposed, suggesting that dark matter was made of baryonic matter, cold dark matter, neutrinos, or an unknown particle yet to be discovered within the theoretical framework of the Standard Model of particle physics.

In spite of this situation, dark matter came to be considered a central theoretical constituent of current astrophysics. In 2003, measurements of the Cosmic Microwave Background Radiation determined the total mass density and the geometry of the universe (Freese 2006). According to this, dark matter accounts for approximately $26 \%$ of the total mass of the universe, whereas $4 \%$ corresponds to the ordinary atoms of chemistry and $70 \%$ to dark energy. Furthermore, in 2006, Clowe et al. published an article entitled "Direct Empirical Proof of the Existence of Dark Matter," which, despite not offering data resulting from direct empirical detection or measurements of any sort, gathered evidence in favour of the reality of dark matter on the basis of weak-lensing observations of two colliding clusters (a cluster merger). These days, most in the astrophysicists' community agree on this: “[ $t$ ]he observed displacement between the bulk of the baryons and the gravitational potential proves the presence of 
dark matter for the most general assumptions regarding the behaviour of gravity" (Clowe et al., 2006, p. L112).

Here is the point I want to make. Those working on LA naturalistic metaphysics may be inclined to construct the postulation of dark matter in terms of an inference to the best explanation as follows:

(P1) Some anomalous phenomena are observed (i.e., gravitational effects, such as light lensing and the curvature of the orbits of galaxies, which cannot be explained by considering current data on the total observable mass of the universe and its expected gravitational effects).

(P2) If the reality of dark matter were postulated, (P1) would be best explained.

Therefore,

(C) There is reason to think dark matter likely exists.

The relevant scientific community accepts the postulation of dark matter as a genuine explanatory assumption given that it responds to certain specific criteria. For instance, astrophysicists assess the possibility of performing successful detections of the entity in question in the course of the investigation. Likewise, they expect to perform measurements of features of dark matter. They also consider the plausibility of predictions that are grounded in the assumption of the reality of this entity, which could not otherwise be made without conditionally assuming its existence. Note yet that astrophysicists have already set forth indirect detections, indirect measurements, indirect experiments and indirect predictions, which overall work on the grounds that at least something like dark matter is in fact entrenched in physical phenomena at a cosmic scale.

The argument above is not intended to contend that inference to the best explanation is inherently metaphysical in character. As an inferential strategy, this form of synthetic argument takes place in scenarios that go from ordinary life situations to highly sophisticated scientific settings. By contrast, the case study aims at showing one way in which LA naturalistic metaphysics enables us to evaluate reasons for granting ontological status to unobservable posits in science. Reasons for granting reality to (at least something like) dark matter appear to suffice. Our conceptual analysis can now give a step further asking the following: do we have similar reasons for positing unobservable entities in metaphysics? 
Allow me to briefly return to Chakravartty's strategy for cashing out the continuity thesis in terms of proximity to scientific investigation (i.e., experiential distance and confirmational risk). I am interested in pointing out a limit of the science-metaphysics continuity. Metaphysicians working in the local trend may appeal to arguments such as inference to the best explanation in order to assess the ontological status of unobservable entities in science. The story above demonstrates that the case of dark matter can indeed be clarified by appealing to metaphysical analysis from a local perspective, namely: reasons are provided in order to judge whether we are in a position to grant reality to an unobservable posit that is still undetectable for our epistemic tools.

Nevertheless, do we need to endorse Chakravartty's contention that science is inherently metaphysical so long us it is interested in unobservable entities? I suggest being cautious in this regard. The fact that both science and metaphysics are interested in the unobservable domain does not entail that we should not distinguish between the unobservables of science and the unobservables of metaphysics, as Chakravartty suggests. The argument above works for the scientific scenario. Perhaps a similar avenue of conceptual analysis may be carried out in view of the positing of unobservable entities in metaphysics, such as universals, and the like. Although I cannot undertake a thorough examination of this avenue here, it seems clear to me that we have better grounds for preliminarily accepting the reality of dark matter than for accepting the reality of, let us say, universals. We work on the basis of the hypothesis that dark matter can potentially be evaluated both in terms of reducing experiential distance and increasing confirmational risk, especially considering the steady theoretical and technological refinements in astrophysics that may in the end lead the community to the construction of crucial experiments. My understanding is that none of this is available for the case of, once again, universals, nor does it need to be presupposed by the standards of rationality of the metaphysics of universals.

\section{Concluding remarks: metaphysics within the scientific image}

This article has examined reasons for granting both GA and LA naturalistic metaphysics a place and role in our overall intellectual endeavour along with the rest of the sciences. The argument relies on a pluralistic stance on our epistemic interactions with reality. Empirical sciences amount to one such interaction, and many shades can be found among them. Similarly, both GA and LA naturalistic metaphysics represent other such instances of epistemic interactions with the 
world. A moral to be derived from our argument is that we should not expect that metaphysics mimics the sciences in order for it to belong to the scientific image. By contrast, both applications of naturalistic metaphysics fulfil their goals insofar as they provide understanding of their respective subject matters (section 2). GA naturalistic metaphysics aids to our understanding of fundamental ontological assumptions involved in scientific disciplines such as quantum mechanics (section 3), as well as LA naturalistic metaphysics provides us with conceptual analyses of the reasons we have to grant reality to unobservable posits in the frontiers of our scientific ontology (section 4). In brief, my hope is that we have gathered sufficient evidence for the claim that both styles of naturalistic metaphysical practice belong to the Sellarsian prospect of the scientific image, contributing to shaping our science-based worldview in various respects.

I am aware that the distinction between GA and LA naturalistic metaphysics is not clear-cut. By contrast, it is evident that the separation between these two branches of metaphysics responds to matters of degrees, which ultimately come down to current developments of scientific research. The examples I mentioned regarding quantum mechanics and dark matter illustrate this point. The former discipline intends to find out about features pervading reality as a whole, and it is only because of this that we call metaphysical reflection on this branch of science GA. By contrast, the investigation of the properties of dark matter seeks to come to grips with a particular scientific entity (or cluster of entities) regardless of the fact that it encompasses a large parcel of reality. It calls, accordingly, for a form of metaphysical theorising that is applied locally to ontological problems arising from this specific posit.

There is yet the Sellarsian question about the putative disciplinary independence of second-order metaphysical examination of first-order scientific disciplines. Recall the distinction between knowing that and knowing how. The sciences deliver a wealth of theories providing knowledge that such and such is the case. Nevertheless, metaphysics is limited to knowing how, where this means "knowing one's way around the scheme of things as they are described by the sciences" (Sellars 1963, pp. 1-2). In this respect, metaphysical reflection unavoidably presupposes a great deal of scientific knowledge: even though philosophers are not expected to be specialist in each and every science, they are expected to know their way around their subject matter.

Here is where we take distance from Sellars' project. On the one hand, we can grant the fact that the various scientific disciplines count as a non-negotiable, primary source of information for metaphysical theorising. Yet, on the other, we 
should still emphasise that even within this view of metaphysics as a secondorder discipline there is still room for defending the disciplinary independence of metaphysical practice in terms of the scope of its positive impact on our understanding of the scientific worldview. This does not mean, as Sellars (1963, p. 4) points out, that scientists have to wait for metaphysicians to clarify their subject-matter, but only that metaphysics has the right to be institutionally established as a discipline that aims at articulating new perspectives on riddles that cannot be fully sorted out in terms of standard empirical research at a given time. This latter, positive impact, I believe, should suffice to convince us that metaphysical practice rightfully responds to the naturalistic turn, thereby belonging to our overall intellectual endeavour.

\section{References}

Bunge, M. "Is Scientific Metaphysics Possible?” The Journal of Philosophy, Vol. 78, No. 17, pp. 507-520, 1972.

ChakravartTy, A. "Metaphysics between the Sciences and Philosophies of Science". In P. D. Magnus and J. Busch (eds.) (2010), pp. 59-77.

"On the Prospects of Naturalized Metaphysics". In D. Ross, J. Ladyman and H. Kincaid (eds.) (2013), pp. 27-50.

Clowe, D., BRADAC, M., GONZÁlEZ, A. H., MARKETEViCH, M., RANDALl, S. W., and ZARITSKY, D. "A Direct Empirical Proof of the Existence of Dark Matter”. The Astrophysical Journal, 648, pp. L109-L113, 2006.

CuMPA, J. "A Materialist Criterion of Fundamentality". American Philosophical Quarterly, Vol. 51, No. 4, pp. 37-42, 2014.

ESFELD, M. "Metaphysics of Science between Metaphysics and Science". Grazer Philosophische Studien, 74, pp. 199-213, 2007.

FREESE, K. "The Dark Side of the Universe". Nuclear Instruments and Methods in Physics Research, A, 559, pp. 337-30, 2006.

HumpHrEYs, P. "Scientific Ontology and Speculative Ontology". In D. Ross, J. Ladyman and H. Kincaid (eds.) (2013), pp. 51-78.

Ladyman, J. "Does Physics Answer Metaphysical Questions?” Royal Institute of Pbilosophy Supplement, Vol. 61, pp. 179-201, 2007.

"Science, Metaphysics and Method". Philosophical Studies, Vol. 160, Issue 1, pp. 31-51, 2012. 
and ROSS, D., with SPURRETT, D. AND COLLIER, J. Every Thing Must Go. Metaphysics Naturalized. Oxford, Oxford University Press, 2007. and "The World in the Data". In D. Ross, J. Ladyman and H. Kincaid (eds.) (2013), pp. 108-150.

Livanios, V. Science and Metaphysics. Exploring the Metaphysics of Properties and Laws. England, Palgrave Macmillan, 2017.

Magnus, P. and Busch, J. (eds.) New waves in the Philosophy of Science. England, Palgrave Macmillan, 2010.

Maudlin, T. The Metaphysics within Physics. Oxford, Oxford University Press, 2007.

Morganti, M. Combining Science and Metaphysics. Contemporary Physics, Conceptual Revision and Common Sense. England, Palgrave Macmillan, 2013.

NeY, A. "Neo-Positivist Metaphysics". Philosophical Studies, 160, pp. 53-78, 2012. Ross, D. "Will Scientific Metaphysics Still Be Philosophy?", Academia.edu http://www.academia.edu/948847/Will_scientific_philosophy_still_be_ philosophy 2012 (Accessed 9 October 2015.)

Ross, D., Ladyman, J., and KInCaID, H. (eds.) Scientific Metaphysics. Oxford, Oxford University Press, 2013.

Sellars, W. Science, Perception, and Reality. California, Ridgeview Publishing Company, 1963.

SoTO, C. "The Current State of the Metaphysics of Science Debate". Philosophica, Vol. 90, No. 1, pp. 23-60, 2015.

"The Epistemic Indispensability Argument". Forthcoming, Journal for the General Philosophy of Science.

VAN FraAssen, B. The Empirical Stance. New Heaven and London, Yale University Press, 2002.

WAtKins, J. W. A. "Metaphysics and the Advancement of Science". British Journal for the Philosophy of Science, 26, pp. 91-121, 1975. 